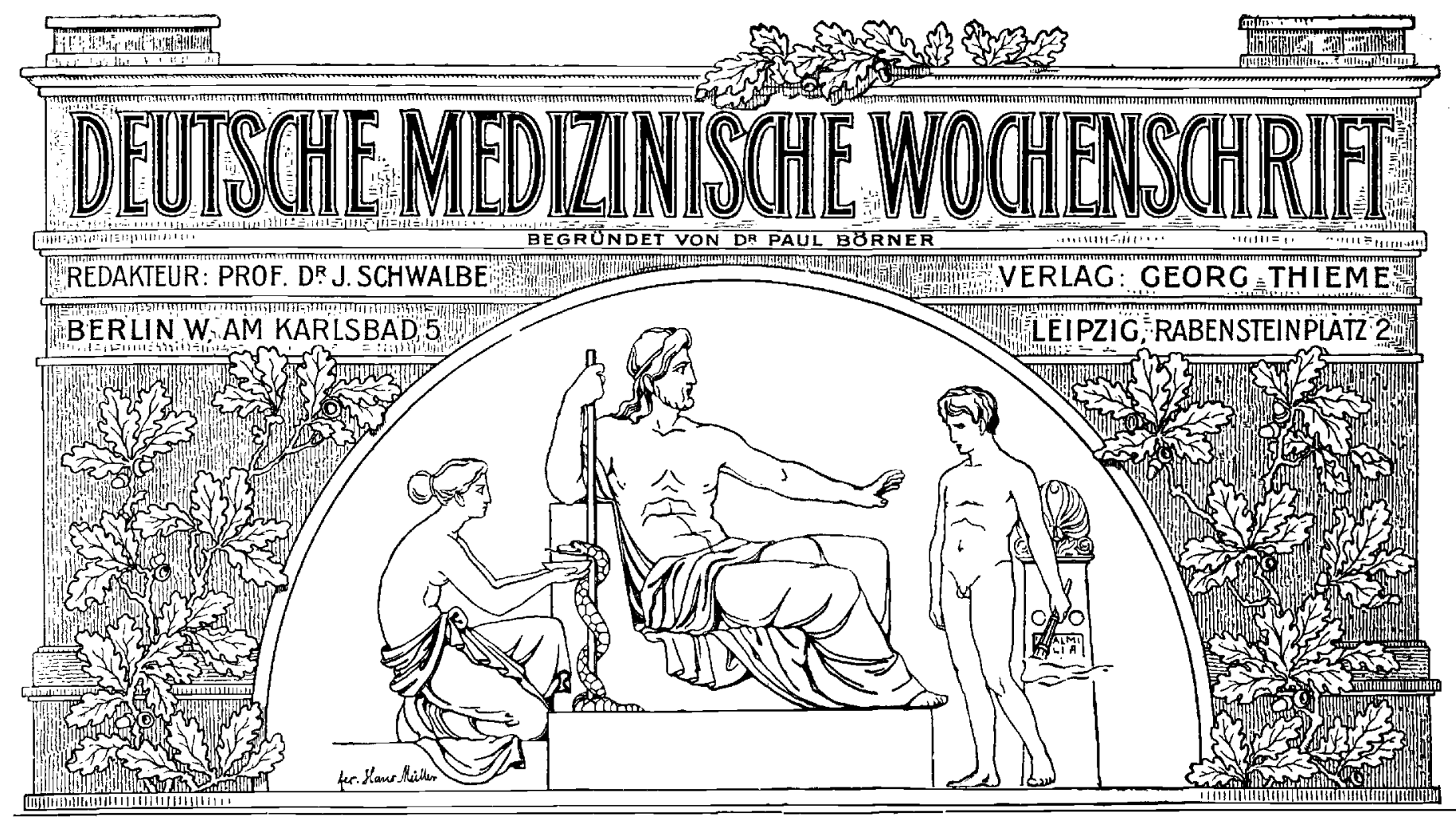

No. 18

Berlin, den 30. April 1908.

34. Jahrgang.

\title{
Aetiologie und Pathogenese der Epityphlitis.
}

\section{Zur Einleitung einer diesen Gegenstand betreffenden Diskussion im Verein für innere Medizin am 30. März 1908.}

\section{Referat, erstattet voll D. v. Hansemann.}

M. H.! Wenıl ich aufgefordert worden bin, über die Patlogenese der Wurmíortsatzentzündung zu sprechen, so möchte ich zunächst einige allgemeine Bemerkungen voranschicken. Ich weiß nicht, ob es nicht vielen von Ihnen ähnlich ergangen ist wie mir bei del Betrachtung der ungeheuer zahllreichen und wichtigen ätiologischen Entdeckuıgen, die in den letzten 25 bis 30 Jahrell gemacht worden sind, llämlich dal Sie bei alledem doch in mallchen Fällen ein gewisses Gefühl des Unbehagens beschlichen liat.

Man hat bei Krankheiten Eunde gemacht in der Form von Bakterien, von Giften, von traumatischen Einwirkungen, anatomiwichen Verhältnissen, die angeboren oder inı späteren Leben er worben waren, und diese als Ursachen von Krankheiten bozeichnet. Sie fanden sich nicht in allen Fällen der Krankheit, sie fanden sich auch gelegentlich bei anderen Zuständen, die mit diesen Krankheitszuständen nicht im Zusammenhang standen, oder fanden sich sogar bei ganz normalen Menschen. Die Entscheidung über die Frage - es bildeten sich bald zwei Parteien, eine Partei dafür, die andere dagegen - wurde nicht immer durch gesicherte wissenschaftliche Tatsachen herbeigeftihrt, sondern sehr häufig durch eine Art von MajoritätsbeschluB. Die Gegner wurden totgeschwiegen oder überstinimt. Dann schien alles in ausgezeichneter Ordnung und vollständig gesichert zu sein, bis sich zach einiger Zeit wieder herausstellte, dal eigentlich doch alles noch in Fluß war und in keiner Weise feststand. Besonders waren es die therapeutischen Resultate, die auf der Basis solcher ätiologischer Forschungen erstrebt wurden, die in keiner Weise dem entsprachen, was man theoretisch hätte erwarten miissen. Meine Herren, ich glaube, daß diesem Zustande ein logischer Denkfehler zugrunde liegt, der die Ursache dafür ist, daß wir sehr häufig von diesen Betrachtungen unbefriedigt sind. Es liegt das nicht an der Art der Entdeckung, sondern an der Art unserer Denkungsweise. Wir machen immer len Fehler, dals wir für eine Krankheit nach der Ursache suchen. Wir müssen uns vielmehr der Denkungsweise nähern, die die exakten W'issenschaften üben, und müssen nicht nach der Ursache fragen, sondern nach den Bedingungen, die notwendig sind, damit eine Krankbeit zustande kommt.

Diese Betrachtungsweise deckt sich nicht ohne weiteres mit dem, was man bisher als Ursache und Disposition bezeichnete, denn dabei hatte man iummer die Neigung, den einen oder andern Faktor speziell als I'rache in den Vordergrund zu schieben. Wenn wir überhaupt den Begriff der Ursache beibehalten wollen, was aber darnach ganz unnötig ist, so würde sich dieser Begriff als eine komplexe Größe darstellen, die aus einer großen Zahl von Einzelbedingungen sich zusammensetzt, wobei auch jede einzelne Bedingung wieder eine komplexe Beschaffenheit haben kann.

Von dieser Betrachtungsweise aus habe ich mich seit vielen Jahren bemüht, immer darauf hinzuweisen, daß es nicht Krankheitsursachen gibt, sondern daß immer eine grobe Reihe von verschiedenen Bedingungen zusammentreffen mub, um eine Krankheit za erzeugen oder eine Krankheit entstehen zu lassen. Es würde sich ganz gewiß lohnen, das für eine große Reihe von Krankheiten auseinanderzusetzen, was ich mir natürlich heute versagen muB. Aber ich will doch diese Art des Gedankenganges einmal anwenden auf das 'Thema, das heute vorliegt, nämlich auf die Epityphlitis.

Wenn wir fragen: welche Bedingungen sind notwendig. damit eine Epityphlitis zustande kommt? so möchte ich zunächst diejenige von den Autoren angeführte hier namhaft machen, der ich den allergeringsten Einfluh auf die Entstehung der Wurmfortsatzentzündung zuschreibe, ich meine die Darmparasiten und die Fremdkörper. Sie wissen ja, daß man behauptet hat, Darmparasiten könnten das machen. Seitdem diese Bellauptung aufgestellt worden ist, habe ich viele Hunderte von Wurmfortsätzen daraufhin durchgesehen. Ich habe im Wurmfortsatz ein einziges Mal beim Menschen einen Oxyuris vermicularis gefunden und einmal beim Orangutang, den ich im Zoologischen Garten seziert habe, einen Trichocephalus dispar. In beiden Fällen war der Wurmfortsatz vollständig gesund. Sonst habe ich Darmparasiten nie darin gefunden. Ich kann also versichern, dą die Darmparasiten jedenfalls für die Entstehung der Wurmfortsatzentzündung keine Rolle spielen.

Dasselbe gilt von den Fremdkörpern. Fremdkörper kommen in den Wurmfortsatz tatsächlich sehr selten hinein, und wenn sie hineinkommen, machen sie noch nicht einmal immer Entzündungen.

Ich habe einen Fall in der Charité seziert, der eine schwer Geisteskranke betraf, die monatelang in der Charité gelegen und nachweislich in dieser Zeit keine Reineclauden zu essen bekommen hatte. Dieselbe hatte im Wurmfortsatz einen Reineclaudenstein, der vermutlich lange darin gelegen hat. Es war nicht eine Spur 
von einer Entzündung des Wurmfortsatzes, auch nicht mikro skopisch. nachzuweisen.

Es ist ganz selbstverständlich. daß perforierende Fremdkörper. wie Nägel. Nadeln. die Erscheinungen der perforieren den Wurmfortsatzentzündung machen können. das spielt aber für das Gros der Erkrankungen keine Rolle. Ich will auch bemerken. daß es perforierende Fremdkörper gibt. die durch den Wurmfortsatz hindurchgehen und trotzdem nicht notwendigerweise Perforationsperitonitis zu machen brauchen. Dafïr besitze ich ein charakteristisches Beispiel.

Es handelt sich da um einen Wurmfortsatz von mittlerer Lănge. der frei flottierend. ohne Verwachsungen. in die Bauchhöhle ragt und an dessen äußerer Seite eine Fischgrăte angewachsen ist. Diese muß notwendig den Wurmfortsatz durchbohrt haben, hat aber nicht die Erscheinungen der Perforationsperitonitis erzeugt. sondern nur eine ganz geringfügige Entziindung. die ausreichte. die Gräte am subperitonealen Gewebe zu fixieren. Das Präparat wurde zufulligerweise bei einem Menschen gefunden. dessen Tod in keinerlei Zusammenhang mit der Yeränderung stand.

Die Fremdkörper können wir für die breitere Praxis der Wurmfortsatzentzündungen meiner Ansicht nach vollkommen ausschliefien.

Das wissen Sie ja. daß es ganz ähnlich ist - ich brauche os nicht ausführlich auseinanderzusetzen - mit den Kotsteinen. Diese sind nicht eine Bedingung für die Entstehung einer Entzündung. sondern sie entwickeln sich durch die Entzündung. Wenn Sie einen wirklichen Kotstein im Wurmfortsatz finden. so können Sie sicher sein. daß die Entzündung schon vorhanden war. Allerdings sind dann die Kotsteine. wie ja bekannt ist. eine Bedingung für die Verschlimmerung der Entzündung. Daß solche Kotsteine durch den Druck schließlich die Perforation herbeiführen. darüber kann kein Zweifel sein. Aber die Entzündung macht der Kotstein zunächst nicht sondern er ist die Folge der Entzündung.

Ich komme nun zu einer andern Bedingung der Wurmfortsatzentzündung. die ich unbedingt für eine Conditio sine qua non halten mub. das ist die Anwesenheit voll pathogenen Bakterien. Eine Wurmfortsatzentzündung ohne pathogene Bakterien kann nicht zustande kommen. Aber wenn inan sich vorstellen wollte. dab das Hineinkommen dieser pathogenen Bakterien in den Wurmfortsatz genügt. um die Entzündung hervorzurufen. so würde man auch damit einen groben lrrtum begehen. Wenn man die Bedeutung der Bakterien für die Entstehung der Wurmfortsatzentzündung betrachten will. so mul man sie in zwoi Gruppen teilen. eine Gruppe. von der man sagen kann. sie sind in bezug auf den Wurmfortsatz ubiquitär. sie sind immer vorhanden und können immer wirken; aber sie wirken nicht immer. sondern nur unter ganz bestimm ten Umständen. Die andere Gruppe von Bakterien wirkt allerdings unmittelbar. wenll sie nur da ist; sie ist jedoch nicht immer da. sie kommt nur ausnahmsweise. unter bestimmten Bedingungen in den Darm und in den Wurmfortsatz hinein. Dahin gehören z. B. die 'Tuberkelbazillen. Dann kann die tuberkulöse Wurmfortsatzentzündung entstehen. Das betrifft die seltenen Fälle von aktinomykotischer Wurmfortsatzentzündung. das betrifft die typhösen Fïlle. und ich glaube, dahin Inüssen wir auch diejenigen Fälle rechnen. die sehr häufig besonders in Frankreich beobachtet worden sind. wo Wurm fortsatztentzündungen in ganz charakteristischer Weise im Anschlup an Influenza aufgetreten sind. und zwar geradezu epidemisch. Was die andern Bakterien betrifft. die große Zahl der literbakterien. vielleicht auch die anä̈roben Bakterien, die ja in der Literatur eine gewisse Rolle spielen. so wirken sie zweifellos nicht ohue weiteres auf den Wurmfortsatz sondern es müssen noch weitere Bedingangen hinzutreten. damit sie zur Wirkung kommen.

M. H.! Bei diesen Bakterien spielt natürlich auch die Jynplatische Einrichtung des Wurmfortsatzes eine gewisse Rolle. Es wird Ihnen allen bekannt sein. daß voll inancher Seite den Follikeln eine ganz besondere Bedeutung bei der Entstehung der Wurmfortsatzentzündung zugeschriebon wird. daß man die Vorstellung gewonnen hat. als wäre der Wurmfortsatz ähulich wie die Tonsillen im Rachen eine Art von Mausefalle, in der die Bakterien abgefangen und nun hier ganz bessonders zur. Wirkung gebracht werden. Ich glaube. dal das in allgemeinen nicht richtig ist. Denn die Follikel. die im Wurmfortsatz vorhanden sind. mub man einmal vergleichend anatomisch betrachten. wie sie bei der Entwicklung des Menschen aus del niederen Tieren entstanden sein könuen. Der Wurmfortsat\% war ja ursprünglich ein sehr erheblicher Bestandteil des Darms, in dem die Zahl der Follikel ebenso wechselte. wie sie in dem übrigen Darm des Menschen wechseln. Sie wissen. daß die Follikel im Darm individuell variieren zwischen 10000 und 80000 . Das ist natürlich in dem ursprünglichen großen Wurmfortsatz. den die Vorfahren des Menschen gehabt haben, auch der Fall gewesen. Bei der Rückbildung sind die spezifischen Teile des Darms geschwunden, die nicht spezifischen sind zusammengerückt. und dadurch imponiert uns der Wurmfortsatz als lymphatisches Organ. Aber Sie müssen bedenken. daf die normale Innenfläche des Wurmfortsatzes von einer gut funktionierenden Schlejmhaut mit Li eberkü hnschen Krypten allsgekleidet ist. die etwas spärlicher sind als im übrigen Darm. daß die Wand eine Muskulatur besitzt. die imstande ist. sich zu kontrahieren und so einen etwaigen Inhalt aus dem Wurmfortsatz wieder herauszubefördern. Eine so unmittelbare Reziehung zur Oberfläche wie an den T'onsillen. wo sich tiefe Lacunen. oft mit einem mangelhaften Epithel bekleidet. in die lymphatischen Follikel hineinsenken. haben die Follikel im Wurmfortsatz keineswegs. Sie verhalten sich genau so wie die Follikel im uibrigen Darm oder in den übrigen Schleimhäuten überhaupt. z. B. auch in der Trachea. in Blasenhals und anderwärts. Es können sich natürlich wie an allen diesen Körperstellen die Entzündungen in den Follikeln lokalisieren, aber alles das läbt sich doch in keiner Weise mit den Tonsillen vergleichell, die gewissermaßen als Wächter ain Tor stehen und alles das abfangen können, was an ihnen vorbei unberechtigterweise in den Körper eindringen will. Ich glaube déswegen. daf die Follikel des Wurmfortsatzes für die kirkrankung durch solche Bakterien, die ich als ubiquitär bezeichmel habe. garnicht in Betracht kommen. wenn sich auch die'dadurch entstehenden Entzündungen in ihnen lokalisieren können und dadurch nitunter besondere Formen annehmen (folliculärer Katarrh. folliculäre Geschwüre). Aber ich schiebe ihnen eine Bedeutung zu für diejenigen Entzündungen. die durch die genannten Bakterien sich entwickeln. die nicht für den Wurmfortsatz als ubiquitär zu gelten haben. und zwar in denjenigen Fällen. wo diese Bakterien nicht vom Darm aus in den Wurmfortsatz gelangen. sondern durch die Blut- und Lymphbahn. Im Wurmfortsatz kommen tatsächlich ebenso lenticuläre tuberkulöse Geschwiilste vor wie im übrigen Darm. die ihren Ausgang von den Follikeln nehmen und denen oin Stadium der intakten Schleimhaut vorangeht. Die Follikel haben viel eher einen zerstörenden Einfluß auf die Bakterien als einen ihre Ansiedelung befördernden.

Nun kommen wir aber zu einer anderen Bedingung. näinlichl der mechanischen, und diese scheint mir für die Wirkung der ubiquitären Bakterien - Sie entschuldigen den Ausdruck. er ist nicht ganz zutreffend. das weil ich. aber ich habe ihn definiert. und Sie werden verstehen. was ich damit meine von ganz besonderer Bedeutung. Ich grïnde meine Behauptung darauf. daß diese Umstände einem allgemeinen pathologischen Gesetz entsprechen. Bs ist ja allgemein bekannt. dal sich in allen Räumen des Körper's Entzündungen bakterieller Natur ganz besonders leicht dann entwickeln und fast mit absolutei Regelmäßjigkeit auftreten, wenn die normale Durchgängigkeit dieser Räume oder die Resorptionsfähigkeit von ihnen aus gestört ist. Wir sehen. dab. wenn die Bronchien vorstopft sind. in den dahinter liegenden Abschnitten Entzündungen auftreten, daß. wenn Polypen oder Schwellungen den Naseneingang verstopfen, chronische Entzüngen in den oberen Luftwegen entstehen. dab. wenn der Pylorus verengert ist. eine Zersetzung im Magen stattfindet. natürlich unter Einwirkung von Bakterien, die auch sonst im Magen vorhanden sind, die aber erst wirken, wenn die Pylorusstenose eintritt. Wir sehen, wenı Steine in der Gallenblase sind, Entzündungen vom Darm. gewöhulich katarrhalische oder dysenterische oder typhöse Entzündungen. in die Gallenblase übergehen und dort Eiter.ungen erzougen. was in der Regel nicht geschieht, wenn keine Gallensteine vorhanden sind. Wir sehen, daß dio Harn- 
blase sehr refraktär gegen Bakterien ist. so lange ein normaler Harnabflub besteht. daß zwar häufig Entzündungen eintreten können. aber bald wieder ausheilen. Ist jedoch der Harnabfluß gestört. bleibt Residualharn zurück. dann sehen Sie. dab die kleinste Spur von Bakterien, die trotz größter Sorgfalt mit dem Katheter oder sonstwie eingeführt wird. bei alten Prostatikern z. B., schließlich schwere. unheilbare Entzündungen erzeugt. Selbst geschlossene Höhlen. wie z. B. das Peritoneum. lassen das erkennen.

Sie können einem Kaninchen in das Peritoneum eine ziemliche (Juantität von Eiterbakterien einspritzen. ohne dals es Peritonitis hekommt: die Bakterien werden einfach resorbiert. Spritzen Sie kurz vorher eine Spur von Jodlösung oder absolutem Alkohol hinrin und erzeugen dadurch eine Herabsetzung der Resorption. so genilgt die kleinste Quantität von Kiterbakterien, um eine tödliche Peritonitis hervorzurufen.

Dadurch entwickelt sich alıch bei der Lebercirrhose und hei anderen Formen des Ascites. die mit Verdickung des Peritoneums einhergehen. wodurch die Resorptionsfähigkeit behindert ist, mit außerordentlicher L,eiclitigkeit eine bakterielle Fintzündung. Werden solche Fälle häüfiger punktiert. so wird die Fntzündung fast immer eitrig. selbst bei größter und sorgPältigster Reinigung und Desinfektion der zur Punktion verwendeten Instrumente.

Das ist das. was wir überall sehen. wohin wir im Körper kommen. Sowie die Resorption resp. der Durchflub. allgemein gesagt. gestört ist. haben sie die Neigung zur bakteriellen Infektion. Die Bakterien können überall dazu kommen. ganz besonders im Darm. wo sie in großen Quantitäten stets vorhanden sind.

Wie liegen nun die Verhältnisse beim Wurmfortsatz? Da haben Sie in der Tat die mechanische Möglichkeit. dab die l)inge im Wurmfortsatz zurückgehalten werden. Es gibt Wurmfortsätze. die ganz frei offen sind. sie gehen trichterförmig aus dem Coecum heraus. Das ist der Zustand. der bei Neugeborenen vorhanden ist und der sich allmählich. bis zum dritten Jahre gewöhnlich. zurückbildet. aber zuweilen als in. fantilistischer Zustand bis in das späteste Leben hinein besteht. Ein solcher Wurmfortsatz ist frei offen. es kann alles hinein. es kann aber auch alles hinaus. Ich habe bisher keinen einzigen Fall von Epityphlitis gesehen. der sich bei einem solchen Wurmfortsatz gebildet hätte. mit Ausnahme der typhösen und tuberkulösen Formen. Die können sich natürlich alch in solchem Wurmfortsatz entwickeln. denn die bedürfen der Bedingungen der Stauung nicht. Meine Herren. eine ganz gewöhnliche Einrichtung ist hier. wie Sie wissen. die Gerlachsche Klappe. Die Gerlachsche Klappe ist nicht immer richtig aufgefaßt worden mit Bezug auf ihre Bedeutung für die Wurmfortsatzentzündung.

Es ist nicht ganz leicht, diese Gerlachsche Klappe richtig zu deuten, und zwar deswegen nicht, weil der Wurmfortsatz keine konstante Lage hat. Sie wissen. der Wurmfortsat $z$ kann nach oben liegen. nach der Leber zu. nach unten, nach dem kleinen Becken 211: er kann sich nach vorn 11mschlagen, er kann hinter dem Darm liegren. alle möglichen Lagen annehmen. er kann lang. er kann kurz sein. er kann eng oder weit sein. Das übt natürlich einen Einfluß auf die Funktion der Klappe aus, wenn der Wurmfortsatz richtig entwickelt ist. wie ihn erwachsene Menschen haben sollen. Sie werden begreifen, daß, wenn der Wurmfortsatz verkehrt liegt. die Klappe 'sich öffnen kann und daß sie auch durch eine andere Lage nach einer andern Richtung sich fester schließen kann als unter normalen Verhältnissen. Ws hat sich durch vielfache Versuche. die ich angestellt habe. herausgestellt, daß eine Klappe. die den Eingang verhindert. sodaß schlecht etwas hineinkommt. im allgemeinen anch den Ausgang verhindert. sodaß auch schlecht etwas heranskommt. Nun kann aber eine solche Vorrichtung, die den Fingangr rerhindert. sich zuxeeilen öffnen und kann sich nach her wieder schließen: denn der Wurmfortsatz liegt nicht konstant. er kann sich bewegen, er verändert seine Lage, und zwar je nach der Füllung des Darmes. Wenn der Darm meteoristisch auf getrieben ist. so erigiert sich auch der Wurmfortsatz. Davon kann man sich leicht überzeugen. Wenn man auf einen leicht meteoristischen Darm in den man auch noch etwas Flüssigkeit hineinfullt, mit der Hand aufdrückt. so kann man die Lage des Wurmfortsatzes dadurch veränderı. Ein Wurmfortsatz. der sich als absolut undurchlässig erweist. öffnet sich z. B. dadurch. Wenn man eine gefärbte Fliissigkeit in das Coecum hineinbringt, die Spitze des Wurmfort- satzes abschneidet und nun auf den Wurnfortsatz drückt, dann entleert sich garnichts. Gebe ich aber etwas Luft hinzu. mache einen künstlichen Meteorismus: sofort erigiert sich der Wurmfortsatz. er wird durchlässig. und die gefärbte Fliissigkeit spritzt aus dem abgeschnittenen Ende hervor. Man sieht also, es gibt Fälle. wo der undurchlässige Wurmfortsat' mit einem $\mathrm{Mal}$ durch die veränderte Sitıation durchlässig wird. Es können Gase hineinkommen. auch natiirlich Kotmassen. die pathogene Bakterien enthalten könnep. Hört die Druckwirkung auf. dann sinkt der Wurmfortsatz wieder in seine ursprüngliche Lage zurlick. er verschließt sich wieder. Jetzt sind die Bakterien in dem abgeschlossenen Sack. und nun sind die Bedingungen gegebel1. wodurch eine Entzundung entstehen kann.

M. H.! Diese Gerlachsche Klappe ist natürlich nicht das einzige. sondern es gibt auch noch andere Dinge. die den Verschluß herbeiführen können; das versteht sich ganz von selbst. Da komme ich nun auf einen Punkt. den man eigentlich früher schon gekannt hat. der aber etwas ominös geworden ist. Man hat ihn eigentlich ganz ausgeschaltet: das ist die Fntzündung des Coecums. Die Entzündung des Coecums spielt meiner Ansicht nach bei der Entstehung der Epityphlitis eine ganz hervorragende Rolle. Allerdings ist in dem Moment. wo man klinisch die Wurmfortsatzentzündung findet, wo sie operativ angegriffen wird. das meist schon längst verschwunden Das ist die eigentliche Typhlitis, die alte stercorale Typhlitis, die entschieden vorkommt und die auf die Entstehung der Wurmfortsatzentzündung einen sehr bedeutenden Einflub ausübt. Wir können das jetzt. nachdem wir die Bedeutung der Wurmfortsatzentzündung kennen gelernt haben. ruhig anerkennen. Es war eine Zeitlang gefährlich. das zu sagen. weil die Bedeutung der Wurmfortsatzentzündung noch nicht in Fleisch und Blut der Aerzte übergegangen war. Das ist jetzt anders geworden. Sie ist uns in Fleisch und Blut übergegangen. und jetzt können wir zur alten Typhlitis zurückkommen. Die Typhlitis existiert. Sie ist eine stercorale. d. h. sie kann entstehen durch Kotansammlung. natürlich auch durch alle möglichen anderen Entzündungserscheinungen. und sie übt einen erheblichen Einflub auf den Wurmforsatz aus. Wenn hier eine Schwellung entsteht. dann braucht gar keine charakteristische Gerlachsche Klappe vorhanden zu sein. Eine mälige Faltenbildung und die Schwellung schliebt den Wurmfortsatz $a b$; sofort sind die Bedingungen gegeben. daf Kotstoffe mit den darin enthaltenen Bakterien retendiert werden. Die Typhlitis kann sich auch bei offenem Wurmfortsatz per contimutatem in demselben fortsetzen. Dann heilt sie aber meist schnell wieder aus. Aus diesen angeführten Bedingungen orklärt sich auch. daf Sie zuweilen bei tuberkulösen Darmveränderungen eine Wurmfortsatzentzündung finden. die ganz typisch der gewöhnlichen Epityphlitis entspricht und nicht tuberkulös ist. Sehr häufig bekomme ich Wurmfortsätze zur Untersuchung von tuberkulösen Menschen; es wird die Vermutung ausgesprochen. sie seien tuberkulös. Sie brauchen nicht tuberkulös zu sein. die tuberkulösen Geschwüre sitzen am Rande. sie verschliefien den Wurmfortsatz. und nun entsteht eine ganz gewöhnliche katarrhalische. folliculäre, eitrige oder nekrotische Epityphlitis. Natürlich in anderen Füllen kommen auch Tuberkelbazillen hinein und machen tuberkulöse Geschwüre.

M. H.! Das ist also die Bedeutung des Verschlusses. der hier eintritt. der wechseln kann. der sich abschließen und wieder öffnen kann und woraus sich die Entstehung der Entzündung mit erklärt. Dieser Verschluß ist eine der Bedingungen für gewisse Fälle von Wurmfortsatzentzündung. und ich möchte glauben, für den größten Teil derselben. namentlich fïr die gewöhnlichen, für die typischen. normalen, wenn ich so sagen darf. Darauf werden wir hingeführt durch eine grobe Reihe von Erfahrungen. die wir bei dem Vorkommen der Wurmfortsatzentzündung gemacht haben. Es ist daraus erklärlich. warum bei ganz jungen Kindern die Wurmfortsatzentzündung sehr selten ist: diese haben gewöhnlich noch keine Klappenbildung. Es begreift sich auch daraus, warum bei alten Leuten die Wurmfortsatzentzündung sehr viel seltener ist als bei jüngeren; denn die Menschen. die eine besondere Disposition durch die Art und Weise ihrer anatomischen Struktur besitzen. eine Wurmfortsatzentzündung zu bekommen 
die haben sie schon bekommen, als sie jünger waren, und bei den Menschen, die älter sind und noch keine gehabt haben, ist eben wegen der Anordnung ihres Wurmfortsatzes die Wahrscheinlichkeit sehr gering, daß sie noch eine Entzündung bekommen. Aber es ist bei älteren Leuten nicht alısgeschlossen und wir sehen auch zuweilen, daß ganz alte Leute Wurmfortsatzentzündung bekommell. Es sind sogar Todesfälle beobachtet worden.

Wir begreifen daraus allch, m. H.!, dab traumatische Bedingungen eine Ursache bilden können. Wenn Sie gesehen haben, daß durch eine Massage, durch einen Druck auf das Coecum die Lage des Wurmfortsatzes sich so ändern kann, da\} ein vorher undurchgängiger Wurmfortsatz durchgängig wird und daß er sich nachher, wenn die mechanische Einwirkung aufgehört hat, wiedel' schließt, so können wir verstehen, wie durch eine mechanische Ursache, durch einen Druck beim Pressen, bei der Defäcation, durch irgendeinen Stob, durch Turnen, Springen, Reiten, Schütteln auf der Eisenbahn etc. solche Verhälinisse eintreten können, wodurch nicht nur Kot mit Bakterien hineingetrieben, sondorn allch ein $\mathrm{Ab}$ schlıb wiederhergestellt wird. M. H.! Die spontan geheilten Wurmfortsatzentzündungen sind ungehener hälufig. Seit vo otwa zwei Monaten die Aufforderıng an mich erging, diesen Vortrag zll halten, habe ich angefangen, solche Dinge zu sammeln, und habe in dieser Zeit neun Fälle zusaminen gebracht bei etwa 180 Sektionen. An diesen geheilten Fällen sieht man, wie der Wurmfortsatz in irgendeiner beliebigen Stellung fixiert ist, und es ist natürlich sehr wohl möglich, daß durch die Art der Fixierung ein neues Moment gegeben ist für das Auftreten einer neuen Entzündung. Aber notwendig ist dies keineswegs.

Es ist ja bekannt, wie auBerordentlich verschiedenartig die Wurmfortsătze gestaltet sind, abgesehen von der verschiedenen Länge und der verschiedenen Lage. Auch diejenigen, die nicht infantilistisch gebildet sind, können eine weite Oeffnung haben, und ich möchte diese schlechtweg als offene Wurmfortsätze be zeichnen. Ich glaube, da 3 man bei diesen im allgemeinen nich viel zu befürchten hat und daßs, wenn nicht besondere Umstände eintreten, ihre Träger vor Wurmfortsatzentzündungen ziemlich ge schützt sind. Die zweite Art von Wurmfortsutzen, die eine Gerlach sche Klappe besitzen, sind dann diejenigen, die zu einer solchen Krankheit disponieren. Aber natürlich ist nicht in jedem solchen Wurmfortsatz eine Entzündung vorhande11, weil auch die iibrigen Bedingungen notwendig. sind, damit dieselbe zustande komme. Unter den in diesen zwei Monaten gesammelten Wurnfortsätzen findet sich auch eine überraschend große Zahl mit frischen, nicht unerheblichen Entzündungserscheinungen (vier Stück), ohne daß sie klinische Erscheinungen gemacht hätten.

Ich möchte glauben, daß das, was sich klinisch als Wurmfortsatzentzündung dokumentiert, und vor allen Dingen die schwereren Formen niemals den Anfang darstellen, sondern dah immer schon eine Entzündung vorangegangen ist, die mehr oder weniger latent verlief. Daher kommt es auch, daf man fast immer an den Wurmfortsätzen, die wegen bedroh licher Erscheinungen exstirpiert wurden, neben den akuten Entziindungen die Spuren alter Entzündungen nachweisen kann

Tch möchte fast die Behauptung aufstellen, die mir wohl von den meisten bestätigt werden wird, daß es nur sehr selten vorkommt, daß ein Mensch im ersten Anfall der Wurmfortsatzentzündung zugrunde geht, sondern erst bei den Rezidiven.

Es gibt noch eine ganze Reihe von anderen Entzündungsformen, die klinisch viel weniger in die Erscheinung treten und meist zufällig bei den Sektionen gefunden werden. Es betrifft das diejenigen Fälle, wo der Wurmfortsatz durch eine ganz ulmsclıriebene Entzündung an der Abgangsstelle in einer Zeit zur Obliteration kommt, wo er zufällig frei von Darm inhalt ist und infolgedessen auch frei von Bakterien. Vielleicht ist es auch möglich, daß unter solchen Bedingungen die darin enthaltenen Bakterien abgetötet und resorbiert werden.
Jedenfalls entwickelt sich bei einem solchen ganz lokalen Verschluß an der Basis des Wurmfortsatzes nicht immer eine eitrige Entzündung. sondern ebenso wie wir das gelegentlich an der Gallenblase sehen, eine sackfölmige Ausdehnung des Wurmfortsatzes mit Sekretstallung, sodaB der Wurmfortsatz in einen Sack umgebildet ist, der mit einer schleimigen, ziemlich klaren Flüssigkeit erfïllt ist. Virchow nannte das dell Hydrops cysticus des Wurmfortsatzes.

M. H.! Das ist wohl im wesentlichen das, was übor die Bedingungell der Wurmfortsatzentzündıng zu sagen ist.

Das alles hat eigentlich für die Praxis gar keine Bedelltung, sondern für die Praxis gibt es nul den einzigen springenden Plinkt - - das ist aber eine Frage, die wir nicht lösen können, sonderı die müssen die Praktiker löselı ... die Frage der Prognose. Meiner Ansicht llach konınit alles einzig und allein darauf an, daß man lernt, die Fälle prognostisch zll $\mathrm{er}$ kennen, und zwar frïhzeitig zll erkennen. Dab es nicht notwendig ist, so viele Wurmfortsätze zu exstirpieren, wie hente exstirpiert werden, ist zweifellos. Es wird eine Menge von Wurmfortsätzen exstirpiert. an denen sehr wenig zll sehen isi. Es besteht da ein Mißverhältnis zwischen denı (hirurgen und dem Anatomen. Die Chirurgen schneiden dell Wurmfortsat: heraus; wir untersuchon ihn und finden in manchen Fiallen gar nichts daran. Dann sagen die Chirurgen: ihr habt falscl untersucht, es ist doch etwas daranl. Nein, meine Herrerl, os ist nichts daran, ganz bestimmt nicht! Ich will allech sagetl. woran es liegt: es liegt daran, daß die Entzündung dann irll Coecum sitzt. Im Coecum ist der Abschlul erfolgt, das cranze macht die Erscheillungen lel Wurmfortsatzentzündung, und wenn Sie den Wurmfortsatz herausschneiden, so ist er ganl normal. Das heilt natürlich aus, ohne weiteres. Ob es so ausgeheilt wäre, ohne die Operation, weiß ich nicht, glanbe es aber; violleicht wäre es auch ohne Operation nicht ausgeheilt. Also die Exstirpation ist an und fïr sich auch in solehen Fällen berechtigt, weil man nicht weiß, was daralls werden kann. Es ist aber auch berechtigt, wenn der Anatoln erklärt, er finde nichts, del Wurmfortsatz sei gesund. Ich meine also, meine Herren, auf die richtige Stellnllg der Prognose kommt es anl, aber es ist nicht Sache des Anatomen, das zll behandeln, sondern Sache des Klinikers.

Ich möchte noch hinzufïgen, dab ich die $\Lambda$ nsicht derjenigen teile, die glauben, daß der Wurmfortsatz im höheren Alter sehr häufig obliteriert ohne Entzündıng. Ich glaube, man kann sehr gut eille solche Involution von einer entzündlichen Obliteration unterscheiden, denn die Involution beginnt stets an der Spitze und schreitet allmählich nach der Basis zil fort, ohne daB in den Wandungen des Wurmfortsatzes oder in der Umgebung entzündliche Veründerungen resp. Adhäsionen auftreten. Die entzündliche Obliteration dagegen beginnt a.l irgendeiner beliebigen Stelle des Wurmfortsatzes, aber mall wird dabei auch inımer makroskopisch oder mikroskopisch die Reste der entzündlichen Veränderung nachweisen können.

Zum SchluB möchte ich noch eine kurze Bemerkung uber die Nomenklatur machen. Ich habe mich gefreut, dal3 der Schriftfïhrer dieser Gesellschaft, Herr Kollege Schwalbe, ohne weiteres auf meinen Vorschlag eingegangen ist, das Thema nicht mit den Worten Appendicltis, sondern Epityphlitis zu versehen. Das leider allgemein iibliche Wort Appendicitis ist eines der fürchterlichsten. das je in der Medizin gebildet wurde. Wenn auch nicht in Berlin erfunden, so hat doch die Berliner Schule leider zur Verbreitung dieses Namens sehr beigetragen. Wenn wir nun nicht ein anderes Wort daftir besäßen, das handlich wäre, so lieBe sich die Anwenduns dieses greulichen Wortes Appendicitis noch entschuldigen. Das ist aber nicht der Full. Herr K üster hat uns das vorzügliche Wort Fpityphlitis geschaffen, und ich sehe keinen Grund ein, wenn nur der gute Wille vorhanden ist, warum wir nicht statt des falsch gebildeten Wortes das richtige anwenden sollten. Ich möchte Sie bitten, daß Sie sich mit mir vereinigen in dem Bestreben, dieses Wort Epityphlitis an Stelle des anderen durehzusetzen.

\section{Referat, erstattet von Geh. Med.-Rat Prof. Dr. Sonnenburg.}

M. H.! Ich kann an Inanches anknüpfen, was uns Herr v. Hansemann mitgeteilt hat, und zunächst sagen, dab auch wir Chirurgen die Appendicitis als eine durch Infektion, speziell durch Bakterien bedingte Entzündung wie viele andere Arten von Entzündung auffassen. Die verschiedenen klinischen Er. scheinungen werden durch die Art der Mikroben, ihre verschiedene Virulenz, durch die Lage und die bestimmten pathologischen Verhältnisse des Wurmfortsatzes durch die wechselnde lokale Empfänglichkeit seines adenoiden Gewebes geniigend erklärt, endlich auch durch die Art der Reaktion des Peritoneums. 
Um aber die Pathogenese und Aetiologie der Appendicitis richtig zu verstehen, muß man nach meiner Ansicht zwischen der Pathogenese und Aetiologie des ersten Anfalls und der folgenden Anfälle unterscheiden und diese voneinander trennen. Nit andern Worten: wir müssen uns zunächst init der Pathogenese der Appendicitis simplex, der katarrhalischen Form der Appendicitis, wie sie auch bezeichnet worden ist, beschäftigen und im Anschluf daran über die Formen der Appendicitis destructiva, die den späteren Stadien der Erkrankung angehören, Erörterungen knüpfen.

Es ist ja von vornherein klar, dab, su lange wir eine Entzündung an einer verhältnismäßig normalen und noch wenig veränderten Appendix haben, eine Infektion dieses Organs nur von außen, vom Darm aus oder durch das Blut entstehen kann. Ist die Appendix aber einmal infolge mehrfacher Anfälle pathologisch verändert, dann hat sie in ihrem Innern oder ihren Wandungen Herde, von denen aus durch irgendwelchen Anlaß, gewissermaßen aus ihr heraus, die Entzündung wieder neu aufflackern kann.

M. H., wenn wir uns also zunächst mit der Appendicitis simplex beschäftigen, so fragen wir nach etwaigen Dispositionen des kleinen Organs zur Entzündung. Wir haben dabei in erster Linie es mit angeborenen Verhältnissen zu tun. Herr v. Hansemann hat Ihnen schon erklärt, in welcher Weise gerade hier Verhältnisse vorliegen, welche als Disposition zur Entzündung angesehen werden müssen. Dazu gehören die abnorme Länge, die verschiedene Lage der Appendix, die Enge ihres Kanals im Verhältnis zu ihrer Länge, der Ansatz und die. Ausbreitung des Mesenteriolums, das ungewöhnlich befestigt oder aber mit wenigen Blutgefäßen versehen sein kann, wodurch oft eine mangelhafte Ernährung bedingt wird; ferner krommt dazll die angeborene Kryptenbildung im Innern, auf die Ashoff aufmerksam macht, der große Follikelreichtum. Das Kindesalter zeigt sich für alle Formen von Infektionen, besonders Darminfektionen geneigter, während das Geschlecht keine Rolle spielt, wohl aber die Art der Ernährung. UnzweckmäBige Ernährung führt zu Darmkatarrhen akuter und chronischer Art. Hierdurch entsteht eine ungünstige Beeinflussung des Wurmfortsatzes. Gerade solche Ernährungsverhältnisse ungünstiger Art muß man zur Erklärung herbeiholen, um auch zu verstehen, daß bei einigen Völkern Appendicitiden häufiger oder seltener als bei anderen beobachtet werden.

So habe ich jetzt bei meiner Anwesenheit in Aegypten von den Kollegen gehört, daß bei den Arabern Appendicitis ungemein selten vorkommt, weil diese sich sehr zweckmäbig und dem Klima entsprechend ernähren. Daran hindert der Umstand nichts, daß der Darm der Araber von Schmarotzern strotzt, von denen man eigentlich voraussetzen muß, daß sie Darmkatarrhe aller Art zur Folge haben.

Derartige anatomische Dispositionen können aber auch fehlen, so z. B. bei Wurmfortsätzen, die kurz, gut ernährt sind und breit in das Coecum einmünden. Das sind Wurmfortsätze, die wenig zu Erkrankungen neigen, da die Gifte, die von außen in sie gelangen, nicht lange haften, sondern leicht wieder zurückbefördert werden können, ehe es zu bleibenden pathologischen Veränderungen kommt.

Nun nimmt jeder Wurmfortsatz mit oder ohne anatomische Dispositionen gern an jeder Art Entzündung des Coecums oder des Darmes teil und dokumentiert diese seine Beteiligung klinisch durch sofort auftretende Druckempfindlichkeit. Diese entsteht zunächst im wesentlichen durch die starke Vermehrung des Sekrets im Blindsack. Die Appendicitis als Teilerscheinung eines Darmkatarrhs ist meiner Ansicht nach so häufig, daß wohl keiner unter uns nicht schon eine Appendicitis in diesem Sinne durchgemacht hätte. Aber nicht nur an den Darmkatarrhen und Darmentzündungen nimmt die Appendix teil, nein - und das ist ein Umstand, auf den wir vielleicht in den letzten Jahren unsere Aufmerksamkeit nicht genügend gerichtet haben - die Appendicitis ist sehr häufig ebenso eine Teilerscheinung einer Allgemeininfektion hämatogener Art.

Die Fälle der sogenannten Appendicitis infectiosa sind bekannt, wo wir im Darm die Hauptstätte dieser septischen Prozesse vorfinden. Die Appendix ist schmerzhafter als der ganze Darm, und wenn wir in solchen Fällen die Appendix entfernen, sind wir erstaunt, verhältnismäfig wenig Veränderungen zu finden; die mikroskopische Untersuchung ergibt aber dann eine vollständige Imbibition der Wandungen mit Bakterien (Streptococcen.)

Das sind Fälle, die wir oft auch nicht durch Operation retten können, die an allgemeiner Sepsis zugrunde gehen. Die allgemeine Sepsis hat sich dann speziell in dem Locus minoris resistentiae, der Appendix, lokalisiert, die durch ihre anatomische Beschaffenheit gewissermaßen dazu prädestiniert ist, während die benachbarten Darmabschnitte sich freier zeigen. Der Patient geht an allgemeiner Sepsis zugrunde, deren Ursprung wir auch bei der Sektion manchmal nicht feststellen können. Ebenso ist oft eine akute Appendicitis als Teilerscheinung septischer allgemeiner Infektion anzusehen, wo dieselbe in Anschluß an eine Phlegmone Osteomyelitis, Furunculosis auftritt. Hier kann man die Appendicitis für einen metastatischen Prozeb halten. Auch hier wiederum ist die Prognose eine sehlechte, da durch Entfernung des einen Krankheitsherdes dem Organismus nicht viel genützt wird.

Anschliebend an diese hämatogene Genese der Wurmfortsatzinfektion kommen nun alle die Formen von Infektionskrankheiten in Betracht, an denen gleichfalls die Appendix sich beteiligen kann und eine Appendicitis entsteht. Ich erinnere an die verschiedenen Formen von Angina. Wie häufig tritt im Verlaufe einer Angina eine akute Appendicitis auf, wie häufig im Verlaufe von Influenza! Das sind Fälle, die uns Chirurgen alle bekannt sind. Bei der Angina sind die verschluckten Eiterund Membranteile, die in der Appendix sich festsetzen und im Blindsack sich weiter entwickeln, bei der Influenza ist es wieder der Darmkatarrh (wie häufig sitzt die primäre Infektion im Darm), an den die Appendicitis sich anschlieBt. Oder es kommt zu einer hämatogenen Infektion der Follikel. Auch der innere Zusammenhang von Rheumatismus und Appendicitis ist bekannt. Wiederum zeigt sich die Mitbeteiligung klinisch dadurch an, daß wir Druckempfindlichkeit nachweisen können. Wir tasten den ganzen Leib ab: er ist am empfindlichsten in der Ileocöcalgegend, eine Appendicitis wird diagnostiziert.

Nun kann sich zweierlei ereignen: Entweder kann infolge günstiger anatomischer Verhältnisse des Wurmfortsatzes die Entleerung der gebildeten giftigen Produkte leicht und ausgiebig in das Coecum und den Darm wieder erfolgen, die entzündlichen Erscheinungen gehen zurück resp. (wenn die Infektion vom Darm aus erfolgt ist) schwinden die Symptome zugleich mit denen des Darms. Oder es überdauert der entzünaliche Zustand der Appendix infolge ungünstiger anatomischer:,Verhältnisse oder Veränderungen pathologischer Art die allgemeine Infektion resp. die spezielle Erkrankung des Darms. Nun geht der entzündliche Prozeb vom Schleimhautlumen aus. Dann haben wir das klinische Bild lokalisierter Appendicitis; das nun die Situation beherrscht. Die Pathogenese derartiger Fälle kann uns dann völlig entgehen, weil die ursprüngliche Ursache nicht mehr nachweisbar. Den springenden Punkt für die Entstehung der Appendicitis bildet die durch besagte Dispositionen entstehende Retention im Blindsack. Es bildet sich ein Passagehindernis entweder dadurch, daß die geschwollene Schleimhaut an einer Stelle das Lumen des Blindsackes verlegt, oder daß durch die erwähnten anatomischen angeborenen Verhältnisse infolge plötzlicher stärkerer Füllung des Organs Drehungen, Knickungen der Appendix entstehen und dadurch Stauung und vermehrte Retention des Sekrets bedingt wird. Die wechselnde Virulenz der Gifte bedingt dann die Intensität der Infektion im Einzelfalle und schafft die verschiedenen entzündlichen Formen, die wir bei der Frühoperation konstatieren. Doch können sich diese einzelnen Formen rasch zurückbilden, je früher und ausgiebiger die Retention beseitigt wird. Klinisch handelt es sich um sogenannte leichte Anfälle.

Wie gestaltet sich nun die Aetiologie und Pathogenese bei denjenigen Formen von Appendicitis, bei denen schon hochgradige Veränderungen pathologischer Art am und im Wurmfortsatz vor dem neuen Anfalle bestehen, verursacht durch längeren Bestand chronischer Entzündung oder hervorgerufen durch aufeinanderfolgende akute Nachschübe, die wieder abgeklungen sind. Ich fasse alle diese Formen unter die sogenannte A ppendicitis destructiva zusammon im Gegensatz zur Appendicitis simplex, von der bisher die Rede war. Es handelt sich hierbei um alle die pathologisehen Veränderungen des Wurmfortsatzes, wie wir sie bei Operationen 
im freien Intervall vorfinden und von denen wir wissen, daß sie gerade die häufigste Ursache der Rezidive abgeben. Da ist einmal die Hypertrophie der Wandung mit Ausbuchtungen im Innern, das Vorkommen vieler Herde mit Mitosen an den nicht erkrankten Stellen, weiter lokale Wandverdünnungen; dazu gehören Kotsteinbildungen ${ }^{1}$ ), Geschwüre, mögen sie frisch oder vernarbt sein, ferner die dadurch bedingten Stenosen mit dahinter liegenden eiterenthaltenden Höhlen, Reste unvollständiger Heilungen nach akuten Anfällen. Durch derartige Veränderungen ist das Organ in seinen Funktionen beeinträchtigt und die geringste Schädlichkeit kann die alten Herde zu neuer Entzündung entfachen. Hier liegt die beständige Gefahr eines akuten Rückfalles vor.

Ganz so wie bei der Appendicitis simplex gehört aber auch hier eine Infektion dazu, um den neuen Anfall auszulösen, aber zum Unterschiede von der ersteren Form gehört dazu nicht immer eine besondere Virulenz der Bakterien, sondern auch weniger virulente Mikroben können auf dem günstigen Nährboden des kranken Organs oder in dem gestauten Sekret pathogene Eigenschaften entwickeln oder wieder erlangen. Schwieriger als bei der Appendicitis simplex werden wir uns über die Pathogenese des neuen Anfalls klar werden können und eine Infektion vom Blut oder Darm aus annehmen. In der Aetiologie dieser Form von Appendicitis spielt aber manchmal das Trauma eine Rolle. Das Trauma, der Stok, kann hier an sich allein eine akute Entzündung hervorrufen, während bei den Formen, wo eine Appendicitis bei einem wenig oder kaum pathologisch veränderten Wurmfortsatz auftritt, das Trauma allein nicht imstande ist, eine akute Entzündung zu bedingen Das Organ ist dann noch viel zu wenig verwachsen, verändert, als daß der Stoß es direkt treffen könnte, und außerdem wird auch, weil hier keine Reste von Entzündungsherden vorhan den sind, das Trauma ohne Bedeutung sein, selbst im Falle Blutungen in der Appendix oder in ihrer Nähe entstehen würden.

Die Appendicitis destructiva wird in sehr verschiedener Weise sich bemerkbar machen. Diese Entzündungen werden zunächst oft zu einer Vermehrung des Inhalts des Wurmfortsatzes führen. Der Inhalt des Wurmfortsatzes kann aber sehr schwer wieder nach dem Coecum entleert werden, einmal.wwegen der Stenosen und Knickungen, dann auch wegen der infolge vorangegangener entzündlicher Prozesse vorhandenen Steifheit des Organs. Die ad maximum gespannten Empyeme platzen. Sodann kommt es zu Perforationen durch Kotsteine und Ge- schwüre mit lokaler und allgemeiner Peritonitis im Gefolge oder zu Gangrän. Das sind weitaus die sogenannten schweren Anfälle.

Ich habe es aus allen diesen Gründen für richtig gehalten, zum besseren Verständnis eine Trennung bei der Aetiologie und Pathogenese der Appendicitis zu machen und zwar zwischen den Formen, wo wir einen nur wenig pathologisch veränderten Wurmfortsatz finden, und den Formen, wo eben schon hochgradige Veränderungen des Wurmfortsatzes Reste akuter Nachschübe vorhanden sind.

Es ist sicher, dab die katarrhalische Entzündung des Wurmfortsatzes nach Beseitigung der Retention ausheilen kann und normale Verhältnisse wieder eintreten können. Patienten, die eine derartige Appendicitis durchgemacht haben, brauchen nicht wieder von neuem zu erkranken. Je kürzer die Retention andauert, je frühzeitiger das Passagehindernis behoben wird, um so sicherer ist dann die völlige Ausheilung. Die Appendix braucht durchaus nicht wieder in einen Entzündungszustand zu geraten. Denn, wenn sie einmal schon auf pathogene Mikroben gewissermafien reagiert hat, so ist es nicht notwendig, dab sie das zweitemal sich in gleicher Weise verhält. Warum im Einzelfalle die Infektion eine akute Appendicitis auslöst, wird uns oft rätselhaft bleiben und nicht zu ergründen sein, wir können nur im allgemeinen sagen, daß besonders virulente Bakterien dazu gehören. Durch welche Umstände aus den Keimen sich Kolonien entwickeln, ist noch ungeklärt. - Erfolgt aber in jenen leichten Fällen eine unvollständige Rückbildung nach Ablauf des akuten Anfalls, so entsteht aus dem akuten Katarrh ein chronischer, und unmerklich, gefördert durch neue entzündliche Nachschübe, entwickeln sich diejenigen Veränderungen, die uns später bei der Appendicitis destructiva in den verschiedensten Formen entgegentreten.

Die Entscheidung darüber, ob im Einzelfalle akuter Erkrankung die Retention durch geeignete Mittel zu beseitigen und damit die Rückbildung zu fördern oder der kranke Herd lieber gleich radikal zu entfernen ist, kann man nur treffen, wenn man auch klinisch die vorliegenden pathologischen $\mathrm{Zu}$ stände voneinander unterscheiden und richtig diagnostizieren kann. Dazu verhilft neben vielen anderen Hilfsmitteln besonders auch die Leukocytose. Diese gibt uns auch über die Intensität der Infektion den besten Aufschluß. Auf solche Weise werden wir imstande sein, im Einzelfalle stets die richtigen therapeutischen Maßnahmen zu treffen.

\section{Referat, erstattet von Fr. Kraus.}

M. H.! Das Thema Epityphlitis begegnet bei den Aerzten schon leicht einem gewissen Taedium. Die neuerliche Diskussion der Aetiologie und Pathogenesis der, wenn nicht alles trügt, an Häufigkeit noch zunehmenden Appendicitis in einem Kreise von Internisten kann auch nur den Zweck haben, eine Grundlage für gewisse prognostische, prophylaktische und therapeutische Ueberlegungen $\mathrm{zu}$ suchen. Irgend otwas Neues, Ermunterndes mub vorliegen. So wie die Dinge jetzt stehen, ist natürlich die Notwendigkeit des chirurgischen Eingreifens zwingend motiviert. Bezüglich der Leistung der internen Appendicitisbehandlung in der Gegenwart möchte ich nur im Vorhinein abermals das Recht der Chirurgen, be der Indikationsstellung $\mathrm{zu}$ entscheiden, betonen und mich dem Di eula a o yschen Ausspruch anschließen: le traitement médical de l'appendicite est nul ou insuffisant. Ein Vergleich der wissenschaftlichen Darstellungen, welche z. B. Nothnagel und S prengel von der Appendicitis geben, mub jedem Unbefangenen eindringlich zeigen, welchen Vorsprung augenblicklich die chirurgische Erfahrung und Forschung auf diesem Gebiete vor der unseren besitzt.

Gleichwohl schließt die Frühoperation ein gewisses intellektuelles Mißbehagen ein. Wir können garnicht anders, als uns praktisch zu ihr zu bekennen, wir büßen es oft genug bitter, wenn wir aus irgendwelchen Gründen uns haben bestimmen lassen, darauf zu verzichten. Aber wir empfehlen die Frühoperation doch nur, weil wir es dem Einzelfall von vornherein nicht anmerken, wie sein Decursus sein wird, und weil wir diesem unberechenbar drohenden Verlauf die ge-

2) Fremdkörper spielen eine ganz untergeordnete Rolle.

ringe Gefahr einer Frühlaparotomie gegenüberstellen können. Aetiologische und pathogenetische Anschauungen, welche die tatsächliche Vielgestaltigkeit der appendicitischen Prozesse aufzuklären geeignet sind, können uns dereinst immerhin therapeutische Anregungen bieten, welche ärztliches Handeln und Unterlassen zu einem rationellen machen.

Da zahlreiche ausgezeichnete und vollständige Zusammenstellungen (bis 1906, ich verweise z. B. auf das Appendicitis. werk von 0. Sprengel) existieren und wir überdies drei Referenten aus verschiedenen Lagern sind, scheint es mir zweckmäßig, die Darlegung des speziell uns in nere Aerzte hier meist Interessierenden in die Aufwerfung, bzw. in die Beantwortung einiger bestimmter Fragen zusammenzupressen.

1. Die Pathogenesis der Appendicitis umfaßt (bei den akuten und chronischen Formen der Entzündung) erstlich die Läsionen des Processus vermiformis selbst, sowie deren Residuen, zweitens die Veränderungen in der Umgebung des Wurmfortsatzes: die abgegrenzten und die diffusen peritonealen oder paraperitonealen Entzündungen. Die erste hier aufzuwerfende Frage geht naturgemä $\mathfrak{B}$ dahin, ob es, ganz abgesehen von Disposition und etwaigen Gelegenheitsursachen, einen wirklich :veranlassenden, einen für sich maßgebenden ätiologischen Faktor der Erkrankung der Appendix selbst gibt? Mit Recht hat man sich in dieser Beziehung bereits daran gewöhnt, (im Sinne von Sprengel) zu unterscheiden zwischen den Bedingungen der Erkrankung, zwischen den Vorgängen am Wurmfortsatz in statu nascendi, der Krankheitsentstehung einer- und der Entwicklung der folgen. den anatomischen Stadien anderseits. Welches also ist diese 
erste Entstehung? Die vorhandenen Methoden, hierüber Auskunft zu erhalten, sind mannigfache. Tatsächlich haben wir bisher am meisten gelernt aus der makroskopischen Besichtigung und noch mehr der kompetenten mikroskopischen Untersuchung der bei den Frühoperationen gewonnenen Präparate. Wichtige Aufschlüsse sind weiter noch zu erwarten von zielbewuften Obduktionen der ganzen Leichen solcher Individuen, welche in gewissen Stadien der Appendicitis gestorben sind. Wertvolle Daten vermag dann immerhin auch eine klinische Untersuchung zu liefern, welche, ausgehend von der Bekanntschaft mit den familiären Erblichkeitsverhältnissen und Dispositionen, sowie der genauesten pathologischen Geschichte des betreffenden Individuums, dessen gesamten Organismus ins Auge faßt und nicht bloß Magen oder Darm oder gar ausschließlich den Wurmfortsatz. Nicht völlig abschließende Ergebnisse hat hier bisher die bakteriologische Forschung geliefert. Doch wird es wenigstens immer wahrscheinlicher, dab nicht blob die bösen, offen das Gepräge der Sepsis tragenden, sondern auch die ganz überwiegende Mehrzahl der klinisch gutartigeren, bei der frischen Operation untersucht, auf Streptococceninfektion beruhen. Sollte sich dies, was bis jetzt allerdings blob als Vermutung hingestellt werden kann (ich verdanke darüber wertvolle Aufschlüsse brieflichen Mitteilungen von $\mathrm{Kretz}$ ), wirklich bestätigen, so läge darin allein schon ein wichtiger Hinweis auf die Pathogenesis. Und was weiter das Tierexperiment betrifft, so steht ihm der Uebelstand entgegen, daß die gewöhnlichen Versuchstiere einen eigentlichen Processus vermiformis nicht besitzen. Immerhin sollte man auch bei uns in Deutschland diesen Forschungsweg (z. B. an Affen) viel mehr betreten, als es bis jetzt der Fall gewesen. Bei der Osteomyelitis, einer hämatogenen Staphylomykose, hat sich die experimentelle Forschung sehr fruchtbar erwiesen. Ganz ohne rein theoretische Rückschlüsse aus den durch alle diese Mittel eruierbaren Tatsachen ist endlich übrigens ein halbwegs umfassendes Urteil über die Vielgestaltigkeit der appendicitischen Prozesse dermalen kaum möglich.

Die Antwort auf die soeben aufgeworfene Frage nach dem veranlassenden Moment und nach der ersten Entstehung der Appendicitis gewinnt, allerdings nur teilweise, mehr und mehr an Bestimmtheit: Die vom Processus vermiformis ausgehenden entzündlichen Prozesse der rechten Darmbeingrube müssen wir uns entstanden denken durch eine Infektion, deren weitere, in Destruktion und Perforation gipfelnde Entwicklung während der verschiedenen anatomischen Stadien durch bestimmte mechanische Faktoren blob mitbestimmt ist. Im allgemeinen darf der Umstand, dab die Appendix ein eigenartiges Organ ist, als verschlimmerndes Moment gelten.

2. Verweilen wir nun zunächst beim Modus, bei den Bedingungen, dem status nascens dieser Infektion selbst.

Nach Roux, Sonnenburg, Karewski, Riedel (bei letzterem mit Zurückgehen auf den histologischen Begriff der Appendicitis granulosa als wesentlich pathogenetischem Faktor) würden sich die appendicitischen Attacken ganz allgemein auf dem Boden eines chronisch veränderten Wurmfortsatzes abspielen, die Appendicitis wäre überhaupt ein chronischer, in Schüben bloß exazerbierender Proze日. Gerade für uns Internisten erscheint auf den ersten Blick eine solche Anschauung sehr verführerisch. Rein klinisch angesehen, zeigen ja sehr viele Appendicitisfälle, besonders beim Erwachsenen, einen eminent ausgeprägten, bisweilen fast ein ganzes Leben umspannenden, chronischen Decursus. Wie uns allen wohl bekannt, gibt es solche chronische Formen, bei derien es gar nie zu typischen Vollattacken kommt. Die spezialistische Praxis wird dabei bald auf den Magen, bald auf den Darm im allgemeinen abgelenkt. Daß der Wurmfortsatz teilnimmt an verschiedenen Erkrankungen der Darmschleimhaut, ist ferner kaum zu bezweifeln. Das Syndrom einer "Enterocolitis" bleibt auch nach gelungener Appendektomie als quälendes Leiden öfter zurück. Nicht selten schließt sich weiterhin der akute Anfall an Indigestionen an etc. Neuere anatomische Untersuchungen beweisen auch in der Tat, dab es von Haus aus schleichende Fälle gibt, z. B. die Beobachtungen von Oberndorfer, sowie die Mitteilungen von M. B. Schmidt über Blutungen in die Follikel des Processus vermiformis, die wahrscheinlich die
Ausdruck leichtester, ohne gröbere Residuen heilender Attacken bei Kindern (mit Schnupfen etc.) darstellen.

Aber die akute Appendicitis befällt doch auch Individuen, welche, bis dahin völlig darmge sund, niemals dyspeptische Beschwerden, appendiculäre Koliken etc. gehabt haben. Kretz z. B. kennt anatomisch manifest akute Erstinfektionen bei intaktem Wurm allerdings nur von Kindern ganz sicher. Ueber das Zahlenverhältnis der völlig akut debutierenden Erstinfektionen erhoffen wir uns von der Berliner Sammelforschung, vielleicht auch von der heutigen Aussprache einige positive Anhaltspunkte. Ferner hat die neuere anatomische Untersuchung allgemein ergeben, daß die schweren destruktiven Prozesse dem Bilde der klinisch akuten Appendicitis entsprechen; in den klinisch chronischen Fällen finden sich ganz gewöhnlich anatomisch leichtere Formen. Eine primäre, progressive, chronisch-interstitielle Entzündung, welche typisch mit Verödung der Drüsen, mit Verlust des Oberflächenepithels verbunden wäre, kann nach dem Urteil der mafgebenden neueren anatomischen Untersucher höchstens nur als etwas Seltenes in Betracht kommen. Der übrigens überhaupt nicht leicht abgrenzbare Begriff der chronischen Appendicitis umfalt somit vor allem Residuen und Regenerationsstadien nach abgelaufenen leichteren akuten Entzündungsprozessen. Eine "physiologische" Obliteration des Wurmfortsatzes lehnen wir nach dem von $\mathrm{Mundt}$ erbrachten Nachweis von Muskelwandnarben wohl alle ab. Der klinische etappenweise Verlauf könnte teilweise übrigens auch in Momenten, die weit entfernt vom Processus vermiformis selbst liegen, begründet sein, z. B. in rekrudeszierenden Infekten anderweitiger Organe. Jedenfalls erwarte ich von dieser Diskussion gerade auch aus speziell internistischen Gesichtspunkten Aeuberungen betreffend das Verhältnis von akuter und chronischer Appendicitis im allgemeinen und speziell in der Richtung der naheliegenden Disponierung zu Attacken durch die letztere.

3. Die Bemühungen, eine traumatische und Fremdkörper-Appendicitis experimentell zu erzeugen, sind vergeblich gewesen. Nach einer brieflichen Mitteilung von Kretz riet Billroth seinem damaligen Assistenten Narath, bei Affen Fremdkörper in den Processus vermiformis einzunähen und über die Anschwellung eine Ligatur anzulegen. Es heilte aber alles reaktionslos ein. Fü rbringer und Sonnenburg haben sich bemüht, den Einfluf von Traumen auf die Entstehung der Appendicitis und ihrer Attacken klinisch zu präzisieren. Ich glaube, Traumen können blob die Bedeutung von Gelegenheitsursachen für die Entwicklung gewisser späterer Teilprozesse im Rahmen z. B. einer residuären Appendicitis beanspruchen, sie machen aber dieselbe nicht im intakten Processus vermiformis eigentlich entstehen. Gutachtlich müßte wenigstens immer der Beginn schwerer Erscheinungen gleich nach dem Unfall maligebend sein. Auch über diesen Punkt werden wir hoffentlich A euberungen zu hören bekommen.

4. In den Mittelpunkt der einschlägigen pathologischen Prozesse wird, entsprechend den wenigstens bei uns in Deutschland zuerst von Internisten vertretenen Anschauungen (Sahli), immer mehr das Verhalten der Lymphfoll ikel gestellt; die Lymphfollikel der Appendix sind das Substrat der Entzündung, die Appendicitis wird in Vergleich gesetzt mit der Angina tonsillaris und der Osteomyelitis. Die Möglichkeit hierzu liegt in der Art der Gefäßversorgung der Schleimhaut. Darin liegt für uns eben etwas Neues, Ermunterndes. Mit dieser Auffassung steht schon ganz allgemein im Einklang, daß es das jugend liche Alter ist, welches für die Epityphlitis prädisponiert: mit der Abnahme der Follikel als Boden der Krankheit schwindet auch die Möglichkeit zur Entzündung. Wenn erfahrungsgemäß allerjüngste Kinder nicht (sehr wenig) befallen werden, erklärt sich dies wohl zum Teil aus dem von v. Hansemann urgierten fötalen Wurmfortsatzursprung, dessen Fortbestehen den Einund Auslauf garantiert. Anderseits, und dies scheint mir viel maßgebender zu sein, fällt auch sonst alles pathologische Geschehen in den Lymphfollikeln überhaupt nicht in das allererste Kindesalter: $70 \%$ der Fälle von adenoiden Vegetationen behandeln wir bei Kindern im Alter von 6-15 Jahren. Unter den Konstitutionsanomalien, welche erfahrungsgemäB als zur Ap- 
pendicitis disponierend angesehen werden müssen, spielt noch am bestimmtesten eine Rolle die exsudative Diathese (Ly m phatis mus). Mit dieser Diathese deckt sich großenteils das, was man in der Praxis unter dem Schlagwort „Erblichkeit" der Appendicitis und der Appendicitis "familiaris“ zu sehen bekommt. Eine Begünstigung der Appendicitis durch Hypertrophie des follikulären Gewebes, sei es auf Grund angeborener Anlage, sei es infolge wiederholter Reizung durch darüber hinweggeschrittene Entzündungen, ist schon nach allem, was wir von den Tonsillen wissen, wahrscheinlich. Aber weit über alles Bisherige hinaus sind es die aus jüngster Zeit stammenden kompetenten mikroskopisch-anatomischen Untersuchungen, welche, gleichzeitig geeignet, auch über den Mechanismus der Infektion weitgehenden Aufschluß zu geben, den ganzen appendicitischen Prozeb der akuten Angina, bzw. dem histologischen Bilde der akuten Tonsillitis an die Seite stellen.

In seinen Untersuchungen über die Topographie der Appendicitis geht Aschoff aus von den Einbuchtungen, in welchen bei normalem Verhalten des Wurmfortsatzes die Epithelschicht durch Lücken der sonst kräftig entwickelten Muscularis mucosae hindurch direkt mit der Submucosa in Berührung steht. Nach Aschoffs (jetzt 600 Fälle und alle Stadien umfassenden) Untersuchungen stellen nun diese Krypten den Sitz der allerersten Veränderung dar: Verlust der Epitheldecke, umschriebene Anhäufung von weißen Blutkörperchen, Fibrinabscheidung im Gewebe und an der Oberfläche; alles dies im sonst histologisch gesunden Wurmfortsatz, ohne Kotstein, ohne Fremdkörper. In den Leukocyten finden sich Gram-positive Coccen und Stäbchen in übergroßer Zahl. Ebenfalls genau den Buchten entsprechend, treten ferner umschriebene Leukocytenanhäufungen in der Muscularis auf, welche zu schneller eitriger Einschmelzung der Muskulatur führen, somit die Bedingungen der Perforation herstellen. Die tiefsten Buchten liegen auf der Seite gegenüber dem Mesenteriolum, und deshalb sollen auch die Perforationen so häufig nach der freien Seite zu fallen. Endlich beginnen auch die pseudomembranösen Schleimhautveränderungen an den Krypten. Somit würde es die topographische Beziehung sein, welche zwischen den leichten und den schweren Formen der Appendicitis mit AbszeBbildung und Diphtherie auch eine Verbindung herstellt. Früher hat Aschoff sowohl die enterogene wie die hämatogene Infektion des Follikelapparates im Wurmfortsatz als möglich hingestellt; jetzt verhält er sich der hämatogenen Appendicitis gegenüber ablehnend. Von höchster praktischer Bedeutung ist, daß eine berechtigte Analogisierung mit der Tonsillarangina auch weitgehende natürliche Heilungs möglichkeiten eröffnet. Eine Tonsillitis kann auch bei mächtigster Schwellung, bei diphtherischen Belägen spurlos zurückgehen. Die anatomische Untersuchung beweist aber auch direkt in der kranken Appendix weitgehende Heilungsvorgänge.

Von noch größerer Tragweite, wiederum gerade vom internistischen Standpunkte, sind, wofern sie sich bestätigen lassen, die in eine andere Richtung weisenden einschlägigen neuesten anatomischen Untersuchungen von $\mathrm{Kr}$ etz. Auch dieser findet in frischen Fällen die schwersten Veränderungen in Buchten zwischen der Schleimhaut. In der abweichenden Erklärung dieser Buchten erblickt nun Kretz für die Entwicklung der Appendicitis den springenden Punkt und stellt eine Reihe mikroskopischer Bilder zusammen, welche ihn einen andern Ausgangspunkt als die Krypte annehmen lassen und ihn ebenfalls zur einheitlichen Auffassung des appendicitischen Prozesses als eines metastatischen führen. Die Reihenfolge dieser Bilder ist folgende. Zuerst Einschwemmung eines pathogenen Organismus, speziell des Streptococcus, auf dem Wege des großen Kreislaufs in die Capillaren, Festsetzen in einer Capillare, zumeist nahe dem Keimzentrum der Lymphfollikel in der Mucosa des Wurmfortsatzes. Aus diesem Keim (vielleicht von Leukocyten transportiert) entsteht eine in den Serienschnitten wurstförmige Kolonie, welche lokal die Gefäßwand zum Absterben bringt; es treten dann massenhaft Blutkörperchen in den Follikeln ein, das Gewebe wird zertrümmert, und schon nach wenigen Stunden erscheinen die Zellen des Keimzentrums ohne Kernfärbung in Auflösung begriffen. Diese infarctähnliche Beschaffenheit der Nekroseherde bei der phlegmonösen Appendicitis, von der man sich in den mikroskopischen Präparaten überzeugen kann, bei sonstiger Unversehrtheit der Schleimhaut, ist in der Tat höchst auffallend. Mit den Blutkörperchen werden auch Mikroorganismen ins Gewebe geschwemmt; sie wandern mit dem Flüssigkeitsstrom, begleitet von Lymphzellen, gegen das Lumen der Schleimhaut und treten durch das Epithel durch. Im Herde gehen innerhalb weniger Stunden wesentliche Veränderungen vor sich. Ohne phagocytäre Reaktion verschwindet die Coccenkolonie (extrazelluläre Auflösung). Die ausgeschwemmten Coccen können mit der Blutung ebenso prompt zugrunde gehen wie die der Kolonie: es folgt Metamorphose der Blutkörperchen, das kranke CapillargefäB kollabiert, es wandern polynukleäre Leukocyten ein etc. Oder es kommt zur AusstoBung des affizierten Follikels, welche von starker Fibrinbildung gefolgt ist. So resultieren trichterförmige, einen Mantel von Lymphoidzellen führende, in der Tiefe die kranke Capillare aufweisende Substanzverluste der Mucosa. Auch hier ist noch Heilung möglich. Wenn die angeschwemmten Mikroorganismen nur teilweise vernichtet werden, kommt es zur Entwicklung des Bildes der metastatischen progredienten phlegmonösen Entzündung, bzw. selbst zur Gangrän des Wurm. fortsatzes; durch Vereiterung der Umgebung nekrotischer Follikel kann Peritonitis entstehen mit und ohne Wandperforation, ebenso auch fortgeleitet von einer Lymphangoitis des Mesenteriolums etc.

Kretz ist ursprünglich auf seine Vorstellung der häma togenen Appendicitis gekommen durch den makroskopischen Befund von gleichzeitig vorhandener Tonsillitis, Lymphdrüsenschwellung bis zur Gegend der Mm. scaleni und akuter phlegmonöser Entzündung des Wurmfortsatzes, sowie durch die Gleichheit der pathogenen Mikroben an diesen Orten. Von der so naheliegenden ursprünglichen Annahme einer enterogenen Verschleppung der Bakterien (verschluckter Mandeleiter) kam er ab, da er Einwanderung der Mikroorganismen auf dem Wege der seitlichen Halslymphknoten in den Angulus Venae jugularis bei seincn Obduktionen nachweisen konnte. Die Metastasierung durch die Blutbahn hatte bereits auch Adrian vor etwa sieben Jahren auf Grund experimenteller Erfahrungen angenommen. Danach erscheint also die Appendicitis als Ausdruck einer A llgemeininfektion. In den jüngsten Tagen teilte Kretz mit, daf nicht blob die Mandeln als Invasionsstellen der hier in Betracht kammenden pyogenen Infektion zu gelten haben. Auf diese Weise gewinnen ältere internistisch-klinische Angaben über die Appendicitis auf allgemein infektiöser Basis ein erneutes Interesse; abgesehen von Angina tonsillaris kommen Rhinitiden, Influenza, Pneumonie, "rheumatische" Affektionen, Gallenblasenerkrankungen (?) u. a. in Erwägung.

Gegen eine hämatogene Entstehung sind zunächst gewisse prinzipielle Einwendungen erhoben worden. Den nicht durch. greifend spezifischen Charakter des Prozesses und die Tatsache, daß frühzeitige Entfernung des Wurmfortsatzes die Krankheit kupiert, darf man wohl kaum im Ernst hier fernerhin anführen. Größlere Schwierigkeiten verursacht schon jeder Versuch eines direkten anatomischen und ebenso auch des klinischen Beweises des hämatogenen Weges selbst: speziell die Untersuchung des Tonsillensekretes und des Blutes ergibt höchstens zweideutige Resultate. Dagegen würde sich die hämatogene Genese zunächst ungezwungen in einen Kreis analoger pathologischer Erfahrungen einfügen. Sie verliert an Sonderbarkeit, wenn wir bedenken, dab auch die Giftdysenterie (Quecksilber, Arsen) nicht enterogen, sondern gleichfalls hämatogen vermittelt wird. Derselbe Umweg des Giftes durch das Blut konnte ferner für den Typhus und für die bazilläre Dysenterie nachgewiesen werden. Dazu käme die Osteomyelitis; die Knocheneiterung wird hier als sekundäre Bakterienniederlassung angesehen, ob man einen primären Herd (Hautwunde, Tonsillarabszeb etc.) finden kann oder nicht. Das sind aber natürlich bloß Analogien, keine Beweise! Nicht blob Adrian konnte übrigens durch Injektion von Bakterienaufschwemmungen in die Ohrvene von Kaninchen folliculäre Entzündung der Appendixschleimhaut hervorrufen. Auch Todesco unter Leitung von Baumgarten hat durch Cocceninjektion in die Tonsille bei Kaninchen eine metastatische Infektion des folliculären Apparates erzeugen können. Eine exklusive Prädilektionsstelle für die Lokalisation von der Blut- 
bahn her ausgelöster entzündlicher Prozesse ist dabei der Processus vermiformis garnicht. Kretz selbst hat bei perakuter Epityphlitis neben Angina im Coecum makroskopisch, im lleum mikroskopisch frische embolische Mykosen in einzelnen Follikeln nachgewiesen. Pick und Berger (Wiener Seruminstitut) fanden anläflich der intravenösen Infektion von Kaninchen mit Typhusbazillenpräparaten Blutungen im Coecum; Kretz konnte das kranke Gefäß im durchbrechenden Follikel demonstrieren.

Bei der groflen Wichtigkeit des Gegenstandes bin ich Herrn Kretz sehr dankbar, dab er mich in den Stand gesetzt hat, dieser Versammlung eine Anzahl von seinen mikroskopischen Präparaten vorzulegen. (Demonstration.)

Zwischen Aschoff und dessen Schülern und Kretz besteht gegenwärtig eine Controverse. Diese zu entscheiden, fällt fortgesetzten mikroskopischen Untersuchungen und Experimenten zu. Die von Kretz ins Auge gefaßten Blutungen können aber nicht leicht im Sinne Aschoffs einfach traumatische sein. Das Operationsverfahren ist nur ein einmaliges, und eine entsprechende Blutung kann natürlich auch nur ganz frisch sein. In den Fällen von Kretz finden sich aber verschieden weit entwickelte Stadien, neben frischer Hämorrhagie mit noch erkennbarem zentralen Bakterienherd auch ältere, solche mit dem, was Kretz „Plättchen“herd nennt. Traumatische Blutungen sind perifolliculär, sie sind durch die Quetschfiguren im Gewebe an den Veränderungen der Umgebung erkennbar. Der umspülende Darminhalt und die vollständig fehlende Reaktion darauf beweist ebenfalls die frische Entstehung inter operationem. Lotheisen hat über. dies einzelne Fälle in situ aufgeschnitten und doch schon die Blutungen gesehen. Sie scheinen schon auch vor der Abtragung durch die Wand durch. Wahrscheinlich bestehen beider, Aschoffs und Kretz', pathogenetische Auffassungen für verschiedene Fälle zu Recht. Die Fälle von Kretz machen teilweise den Eindruck besonderer Schwere. Ob das Gros enterogen oder hämatogen ist, läßt sich heute natürlich nicht entscheiden. Aber ich hoffe, daB sich unsere Diskussion auch auf den folliculären Beginn und Charakter des appendicitischen Prozesses überhaupt und die Möglichkeit enterogener und hämatogener Entstehung erstreckt.

Mag ein enterogener folliculärer Beginn oder eine bakteriämische Metastasierung angenommen werden, in jedem Fall orhebt sich die theoretische Frage nach dem Grunde des Haft enbleibens der Mikroorganismen. Hier müssen wir uns vorläufig mit Hypothesen behelfen. Die Ablagerung der Bakterien im Knochenmark (dieses enthält solche bekanntlich schon bei geringfügigen örtlichen Infektionen) kann als Schutzvorkehrung angesehen werden; das Knochenmark gilt wohl mit Recht bei vielen Infektionen als Entwicklungsstätte der spezifischen Immunkörper. Die Funktionen des lymphatischen Systems in Lymphknoten, Follikeln etc. sind uns offenbar nur zum geringsten Teil bekannt, eine bakterizide Fähigkeit ist auch hier wenigstens wahrscheinlich. Aber die lokale Empfänglichkeit des adenoiden Gewebes des Wurmfortsatzes mul unbedingt als eine wechselnde angenommen werden, wenn wir überhaupt versuchen, die Vielgestaltigkeit der appendicitischen Prozesse zu verstehen. Es ist sehr bedauerlich, dab wir diagnostisch garnichts über Entwicklung und Leistungsfähigkeit des Darmlymphgewebes anzugeben vermögen, ebenso wie wir über die so verschiedene Resistenz des Peritoneums gegenüber den Entzündungserregern im Einzelfall gar kein Urteil besitzen. Wir besitzen nicht einmal ein abgeschlossenes Verständnis der tonsillogenen Bakteriämien im allgemeinen.

5. Wir haben oben gesehen, daß die Entwicklung der Appendicitis in den verschiedenen Phasen mitbestimmt ist durch mechanische Momente. Der Verlauf hängt nicht blob von der ursprünglichen Heftigkeit der Entzündung, der Virulenz der Infektionserreger und der Beschaffenheit des lymphadenoiden Gewebes der Appendix ab, sondern auch von Faktoren, welche sich im allgemeinen dahin definieren lassen, dab die akut entzündlichen Erscheinungen durch Retention im Wurmfortsatz hinter einem Passagehindernis verschlimmert werden und dab die Gefäbversorgung des Proc. vermiformis dürftig ist. Talamon führte hier bekanntlich vor allem den
Kotstein an, Lieulafoy die Cavité close, nicht bloß infolge von Kotsteinen oder Narbenstriktur, sondern auch durch eine das Lumen obturierende Verschwellung. Sprengel erblickt die im allgemeinen nicht zu leugnende Rolle des Kotsteins speziell darin, dab seine Anwesenheit die akute Verschwellung nicht rückgängig werden läBt. Ich hoffe, dab auch in betreff aller wichtigeren einzelnen mechanischen Faktoren anregende Aeußerungen in unserer Diskussion erfolgen werden. Ich denke dabei auch an die Enge dieses Muskelrohrs mit seinem großen Follikelreichtum an und für sich, die leichte Möglichkeit des Durchpressens von Entzündungsprodukten aus den affizierten Follikeln gegen die Serosa zu, die Bedeutung von natürlich zustandekommender, also lähmungsartiger oder medikamentös (Opium) herbeigefiuhrter Erschlaffung des Muskelrohrs und deren Einfluß auf die LymphgefäBinfektion (Peritonitisgefahr) u. a.

Dies sind die Fragen, auf die wir uns heute nach meiner Meinung zunächst und vorwiegend verlegen sollten, damit die Diskussion nicht in der Breite zerfliebt. Ausgegangen bin ich von der Absicht, eine Basis zu gewinnen für therapeutische Ueberlegungen. Nun, es ist bereits anatomisch festgestellt, daf Appendices, welche schwerste (diphtherische) Formen der Entzündung zeigen, verhältnismäßig oft ausheilen. Die Befunde von Kretz sind geeignet, den therapeutischen Bestrebungen einen noch viel weiteren Umfang anzuweisen. Speziell auch uns Internisten eröffnen sich wieder gewisse Aussichten. Vorläufig kann allerdings die anatomische Erfahrung dem chirurgischen Standpunkt der Frühoperation noch nicht entgegengestellt werden. Wir können glücklicherweise die einschlägigen Untersuchungen über eine auf die Pathogenesis aufgebaute Behandlung in aller Muße fortsetzen, da die heutige chirurgische Therapie ohnehin bereits ganz Ausgezeichnetes leistet.

Zusammenfassung. Es wird sonach vorgeschlagen, speziell folgende Fragen zu diskutieren:

1. Gibt es, abgesehen von Disposition und Gelegenheits:ursachen, einen für sich maßgebenden Faktor der Erkrankung der Appendix im besprochenen Sinn?

Der Infekt als veranlassendes Moment. Relation zwischen diesem und den mechanischen Faktoren, welche die weitere Entwicklung mitbestimmen, im allgemeinen.

2. Akutes Debut der Appendicitis. Beziehung zwischen chronischer Appendicitis und den Attacken überhaupt.

3. Trauma, Fremdkörper. Sind sie mehr als Gelegenheitsursachen?

4. Verhalten der Lymphfollikel der Appendix im appendicitischen ProzeB. Analogie der Appendicitis mit Angina und Osteomyelitis. Untersuchungen von Aschoff und Kretz: Worin liegt die Bedeutung dieser Arbeiten und Auffassungen gerade für die innere Medizin, $d$. h. für unsere Auffassung der Tragweite der natürlichen Heilungstendenz, als Grundlage für prognostische und therapeutische Gedanken?

5. Welche spezielle Bedeutung haben bestimmte mechanische Momente: Kotstein, Vase close, akute Verschwellung, lähmungsartige Dilatation des Muskelrohrs etc.?

6. Theoretische Bedeutung der Virulenz der Mikroben sowie der Resistenz und der Funktionsfähigkeit des lymphatischen Apparates überhaupt und im Darm, der Widerstandskraft des Peritoneums? 